\title{
Mecanismos de transferencia de tecnología como elementos del fortalecimiento del conocimiento acumulado en la industria biofarmacéutica mexicana: El Caso de la UDIBI - IPN \\ Mechanisms of technology transfer as elements of the strengthening of accumulated knowledge in the Mexican biopharmaceutical industry: The Case of the UDIBI - IPN
}

Ana Lilia Sánchez Regla ${ }^{1}$, Alexandra Lizeth Ortiz Guzmán, María del Pilar Monserrat Pérez Hernández ${ }^{2}$, Igor Rivera ${ }^{3}$ y Sonia Mayra Pérez Tapia ${ }^{4}$

Palabras clave: transferencia de tecnología; mecanismos de transferencia de tecnología; biotecnología; industria biofarmacéutica Keywords: technology transfer; technology transfer mechanisms; biotechnology; biopharmaceutical industry

Recepción: 13-08-2018 / Aceptación: 18-01-2019

\section{Resumen}

Introducción: La literatura relacionada con la transferencia de tecnología (TT), ha colocado un mayor énfasis en las organizaciones una vez que han establecido un vínculo de colaboración con instituciones de educación superior (IES); sin embargo, existe escaso conocimiento que observe el efecto inverso, es decir, la TT al interior de una IES tras esta interacción. Este trabajo aborda el caso de un laboratorio de investigación y desarrollo (I\&D) integrado a una universidad pública federal, que, además de generar ciencia básica para proyectos internos, ofrece servicios tecnológicos al sector biofarmacéutico nacional e internacional; cuyos ingresos, le permiten autofinanciar su operación, empleando de forma marginal el presupuesto federal. Bajo un enfoque organizacional e institucional, este artículo tiene como objetivo dar a conocer los mecanismos de TT que confluyen en este laboratorio, pero también, exponer la contribución de estos al fortalecimiento de su conocimiento acumulado.

Método: Este estudio se realizó a partir de veintiocho entrevistas semiestructuradas con personal y agentes externos de la UDIBI, empleando cuestionarios diferenciados con preguntas abiertas, y otras, cerradas con escalas tipo Likert. De igual manera, fueron revisados diferentes convenios de colaboración sostenidos entre este laboratorio y otros actores de la industria con la finalidad de observar el objeto de su interrelación. El procesamiento de la información fue realizado empleado el software NVIVO, lo que favoreció a realizar un análisis más eficiente.

\footnotetext{
${ }^{1}$ Instituto Politécnico Nacional, México. E-mail: alsr8888@gmail.com

${ }^{2}$ Instituto Politécnico Nacional, México, Centro de Investigaciones Económicas, Administrativas y Sociales

${ }^{3}$ Instituto Politécnico Nacional, México, Unidad Profesional Interdisciplinaria de Ingeniería y Ciencias Sociales y Administrativas

${ }^{4}$ Instituto Politécnico Nacional, Unidad de Desarrollo e Investigación en Bioprocesos

(C) Universidad De La Salle Bajío (México)
} 
Resultados. El laboratorio institucional estudiado requiere allegarse de conocimiento de última generación para desarrollar su operación crítica basada en la generación de ciencia básica y la oferta de servicios tecnológicos. Se encontró que al interior de esta entidad politécnica confluyen múltiples y variados mecanismos de TT (organizacionales como institucionales), cuyos productos se traducen en conocimiento de diversa naturaleza que complementa y potencia la base acumulada de conocimiento propio y es base para la explotación de productos científicos, tecnológicos y servicios de alto valor tanto para la academia como para la industria. Otro hallazgo identificado fue que no sólo estos mecanismos transfieren tecnología en un solo sentido; sino, que dada la contribución que hace este laboratorio a su fuente de conocimiento, el flujo se convierte en bidireccional.

Discusión o Conclusión: Los diferentes mecanismos aquí presentados son el producto de la dinámica de la interacción que sostiene el laboratorio estudiado con actores externos e internos, donde, el conocimiento asimilado de estos, junto con el conocimiento propio; pero al mismo tiempo de una visión científica-comercial por parte de su directiva. Todos en conjunto, conforman una importante base de su actual desarrollo, expansión, e incluso, su autosostenimiento; representando así, uno de los escasos casos de excepción en México, de unidades científicas similares integradas a IES públicas que aspiran a trascender hacia un enfoque emprendedor de las universidades.

\begin{abstract}
Introduction: The literature related to technology transfer (TT), has placed greater emphasis on organizations once they have established a collaborative link with higher education institutions (HEIs); however, there is little knowledge to observe the inverse effect, that is, the TT within an HEI after this interaction. This work deals with the case of a research and development laboratory ( $\mathrm{R} \& \mathrm{D}$ ) integrated to a federal public university, which, in addition to generating basic science for internal projects, offers technological services to the national and international biopharmaceutical sector; whose income allows them to self-finance their operation, using marginally the federal budget. Under an organizational and institutional approach, this article aims to publicize the mechanisms of TT that come together in this laboratory, but also, expose the contribution of these to the strengthening of their accumulated knowledge.
\end{abstract}


Method: This study was conducted from twenty-eight semi-structured interviews with personnel and external agents of the UDIBI, using differentiated questionnaires with open questions, and others, closed with Likert scales. Likewise, different collaboration agreements between this laboratory and other industry players were reviewed in order to observe the purpose of their interrelation. The processing of the information was done using the NVIVO software, which favored a more efficient analysis.

Results: The institutional laboratory studied requires the gathering of latest generation knowledge to develop its critical operation based on the generation of basic science and the offer of technological services. It was found that multiple and varied TT mechanisms (organizational and institutional) converge within this polytechnic entity, whose products are translated into knowledge of diverse nature that complements and enhances the accumulated base of our own knowledge and is the basis for the exploitation of scientific products. , technological and services of high value for both academia and industry. Another finding identified was that not only do these mechanisms transfer technology in only one direction; rather, given the contribution that this laboratory makes to its source of knowledge, the flow becomes bidirectional.

Discussion or Conclusion: The different mechanisms presented here are the product of the dynamics of the interaction that the laboratory studied with external and internal actors, where the assimilated knowledge of these, together with the own knowledge; but at the same time of a scientific-commercial vision on the part of its directive. All together, they form an important base of their current development, expansion, and even self-sustainability; representing, thus, one of the few cases of exception in Mexico, of similar scientific units integrated to public HEIs that aspire to transcend towards an enterprising approach of the universities.

\section{$\underline{\text { Introducción }}$}

La capacidad innovadora de las organizaciones, particularmente las de alta intensidad tecnológica, no puede sostenerse de la exclusividad de los recursos y habilidades propios; sino que, éstas requieren fuentes de conocimiento externo para crear productos innovadores, bienes y servicios (Arora et al., 2004). En este sentido, la transferencia de tecnología representa para las organizaciones un proceso crítico (Cohen \& Levinthal, 1990), lo que la convierte en un componente clave en el proceso de innovación de múltiples organizaciones (Lichtenthaler y 
Lichtenthaler, 2010); incluso, su alcance, puede verse reflejado en la obtención de ventajas competitivas como pueden ser la experiencia, costos laborales y acceso a los mercados (Argote et al., 2000).

Dentro de la literatura relacionada a los mecanismos de transferencia de tecnología pueden encontrarse dos enfoques teóricos, uno orientado a los canales de transferencia ocurridos a nivel organizacional (Bozeman, 2000; Szuslanki, 2006; Tsai, 2001), y un segundo, los que se generan al interior de instituciones desarrolladoras de investigación y desarrollo -entiéndase universidades o centros de investigación, institutos científicos, etc.- (Cohen et al., 2001; Rosenberg y Nelson, 1994, D’Este y Patel, 2007).

Los estudios empíricos sobre la TTgenerada dentro de las organizaciones se desarrollan en una amplia gama de líneas de análisis tales como: i) la transferencia de tecnología en diversos sectores industriales (Powell, W., et al., 1996; Fukugawa,N.,2016), ii) la competitividad de las organizaciones (Mowery \& Oxley, 1995; Szuslanki,1996), iii) aprendizaje organizacional (Levitt \& March, 1988), iii) empresas spin off, iv) contratos de I\&D y las patentes como parte de las estrategias tecnológicas (Berbegal- Mirabent, et al., 2017); v) políticas públicas (Bozeman, 2000); vi) productividad y eficiencia de mecanismos de TT (Hu, et al., 2005).

De forma más específica, para la industria farmacéutica y la biofarmacéutica, distintos actores han analizado factores como la creación de organizaciones spill-overs y el impacto que éstas tienen en estas industrias en diversos países (Bianchi et al., 2001; Audrestch, 2001; Audrestch y Feldman, 2003); licenciamiento como fuente de comercialización de tecnología (Mowery y Oxley, 1995; Cohen y Levin, 1989; Powell, 1998); alianzas estratégicas (Chesbrough y Crowther, 2006; Teece \& et al., 1997; Rothaermel, 2001), entre otras líneas más.

En contraste, los trabajos empíricos desarrollados sobre la TT en las instituciones generadoras de I\&D, de forma general parten del planteamiento de que éstas son un importante catalizador de innovaciones hacia las organizaciones (Etzkowitz \& Leydesdorff, 2000), y tienen un relevante rol en el avance y desarrollo científico, tecnológico de los países, sus sectores industriales e incluso en la sociedad (Cohen et al., 2002; Rosenberg y Nelson, 1994, D'Este y Patel, 2007). Considerando este argumento, se han derivado análisis sobre los mecanismos de transferencia en el sentido de revisar su eficiencia al interior de la instituciones (Debackere \& Veugelers, 2005; Bozeman, 2000; Bozeman \& et al., 2015), la relación universidad e industria a partir de sus mecanismos de transferencia (Etzkowitz \& Leydesdorff, 2000; Etzkowitz, 2003; 
Perkmann \& Walsh, 2007); los vínculos de innovación científicos, la labor tanto de grupos de investigación y la interacción de estos en la industria. tzkowitz, 2003 y Patel, 2005), o bien, la relevancia e impacto de las patentes (Etzkowitz \& et al., 2000; Heller \& y Eisenberg, 1998; Levin, et al., 1987) Klevorick, Nelson, \& Winter, 1987) (Debackere \& Veugelers, 2005) (Bozeman, Technology transfer and public policy: a review of research and theory, 2000) (Bozeman \& et al., 2015) (Etzkowitz \& Leydesdorff, 2000) (Perkmann \& Walsh, 2007) (Etzkowitz, 2003) (Etzkowitz, 2003) (Heller \& y Eisenberg, 1998) (Etzkowitz \& et al., 2000) (Levin, Klevorick, Nelson, \& Winter, 1987).

Como puede apreciarse, existen múltiples evidencias empíricas relacionadas con la transferencia de tecnología, tanto en el ámbito organizacional como en el académico, por tanto, se consideró apropiado contribuir a la expansión de la literatura exponiendo, bajo estos dos enfoques, los mecanismos de transferencia de tecnología identificados en un laboratorio científico integrado a una IES pública en México, que, por un lado, mantiene la misión institucional de generar ciencia básica y formar estudiantes; mientras que, por el otro, brinda servicios tecnológicos a la biofarmacéutica nacional e internacional.

El laboratorio científico al cual se hace la referencia es la Unidad de Desarrollo e Investigación en Bioprocesos (UDIBI), mismo que está integrado a la estructura de la Unidad de Desarrollo e Innovación Médica y Biotecnológica (UDIMEB) perteneciente a la Escuela Nacional de Ciencias Biológicas (ENCB), dependencia académica del Instituto Politécnico Nacional (IPN).

La UDIBI fue creada en 2009 con el propósito de desarrollar la investigación y el desarrollo que respalda el "Factor de Transferencia" o Transferón ${ }^{\circledR}$, como se le conoce de forma comercial. Este biofármaco es producto desarrollado por científicos politécnicos y está patentado por el IPN y está indicado para el tratamiento enfermedades relacionadas con alteraciones en el sistema inmunológico, como alergias, autoinmunidades, inmunodeficiencias e incluso cáncer (UDIMEB-IPN, s.f.).

Desde sus inicios, la UDIBI ha desarrollado un conjunto de capacidades tecnológicas que le han servido para ser un referente científico en el ámbito académico, pero también, para ser un actor con una posición plenamente identificada en la cadena de valor de las IF y IBF, debido a la alta competencia de su personal y la calidad de sus servicios tecnológicos. 
El desarrollo de dichas capacidades tecnológicas de este laboratorio institucional ha sido paulatino y sostenido; y su evolución, considerando sus propias particularidades, ha transcurrido bajo los siguientes planos:

a) Creación: Contando con una base de experiencia, y bajo la infraestructura de la UDIMEB, la creación de la UDIBI se derivó principalmente de una necesidad específica y, a partir de ahí, se realizaron estudios de viabilidad, la determinación de su objetivo, misión, visión, requerimientos de personal y estándar científico, identificación y requerimientos de equipos especializados e infraestructura.

b) Desarrollo: Esta etapa se caracterizó por el diseño e implementación de procesos de organización y condiciones técnicas para la ejecución de manuales de buenas prácticas de laboratorio y el establecimiento de un sistema de calidad. Asimismo, se desarrolló un programa de capacitación interna y externa para asistir a cursos y a congresos nacionales e internacionales de prestigiosas escuelas en disciplinas como inmunología, farmacología, etc.; asimismo, se abrieron las puertas para la incorporación de estudiantes para el desarrollo de proyectos de investigación y colaboración con proyectos internos de I\&D (Factor de Transferencia).

c) Expansión: Contando con una plataforma de conocimiento e infraestructura, la UDIBI identificó la existencia de necesidades en el SFB que no estaban siendo satisfechas, o bien, se estaban desarrollándose bajo niveles de calidad inferiores. Para este fin, abrió una línea de servicios tecnológicos, contrató un mayor número de personal y obtuvo la acreditación por COFEPRIS $^{1}$ para constituirse como un Laboratorio Tercero Autorizado (LTA), y efectuar a partir de ahí, pruebas de biocomparabilidad y estudios preclínicos y clínicos. De forma posterior, las necesidades de desarrollo y crecimiento requirieron la vinculación con otros actores del sector, estableciendo relaciones de colaboración inter-organizacional con empresas biotecnológicas internacionales para el desarrollo de otros proyectos de I\&D.

Considerando estos antecedentes, y por el rol que ejerce el laboratorio aquí estudiado en la cadena de valor del sector de biotecnología en México, permite identificarlo como una

\footnotetext{
${ }^{1}$ La Comisión Federal para Prevención de Riesgos Sanitarios (COFEPRIS) es el organismo rector en materia de regulación sanitaria del Gobierno de la República y es responsable del ejercicio de las atribuciones en materia de regulación, control y fomento sanitarios en los términos de la Ley General de Salud.
} 
Mecanismos de transferencia de tecnología como elementos del fortalecimiento del conocimiento acumulado en la industria biofarmacéutica mexicana: El Caso de la UDIBI - IPN

organización intermedia $(\mathrm{OI})^{2}$, ya que funge como un puente al alinear las necesidades de éstas con los lineamientos sanitarios de medicamentos biotecnológicos que exige la autoridad gubernamental ${ }^{3}$.

Asimismo, por las características de su operación, la UDIBI se relaciona con la naturaleza de la I\&D y la actividad innovadora de la industria farmacéutica mexicana, ya que como lo señala Guzmán (2014), éstas requieren de fuentes externas para allegarse de conocimiento obteniéndolo de distintos mecanismos. Bajo esta hipótesis, y dado que, la primera mantiene una intensa interacción, tanto con unidades científicas al interior del IPN, como también, con actores privados de la biofarmacéutica, el artículo que se presenta tiene como propósito exponer, desde los enfoques organizacional e institucional, los distintos mecanismos de transferencia de tecnología que en ella confluyen y su aportación al conocimiento de la unidad objeto de estudio.

En este sentido, la aportación esperada de este artículo, es la de integrar a la vasta línea de análisis vertidos sobre la transferencia de tecnología y sus diferentes mecanismos, dando a conocer el caso de estudio de un laboratorio científico integrado a una IES pública, cuyas características representan una excepción en el contexto de la educación superior pública en México; ya que por una parte, desarrolla su misión institucional, y por la otra, comercializa su I\&D lo que le permite autosustentar su operación, y de esta forma, no depender del presupuesto otorgado por gobierno federal.

La estructura que guarda este artículo está asentada en cuatro apartados. El primero de ellos, recopila, bajo los enfoques institucional y organizacional, elementos teóricos específicos relacionados a la transferencia de tecnología, como: principales conceptos, motivos para transferir tecnología y diversos tipos de mecanismos de TT. El segundo apartado, describe la metodología desarrollada para la obtención de información relacionada a este caso de estudio. El tercer capítulo presenta como hallazgo los distintos mecanismos de TT identificados al interior

\footnotetext{
${ }^{2}$ Una Organización intermedia puede identificarse como aquellos agentes, brokers o enlaces que facilitan la actividad innovadora entre dos o más partes, fungiendo como puente entre los generadores y los usuarios de tecnologías asumiendo distintas funciones con el objetivo de introducir al mercado nuevos productos, procesos, métodos organizacionales o comerciales (Pérez, 2014).

${ }^{3}$ El artículo 222 Bis, de la Ley General de Salud establece el régimen legal de los medicamentos biotecnológicos en México y prevé que todos los medicamentos biotecnológicos deben obtener el registro sanitario, siempre y cuando cumplan con los requisitos y pruebas que demuestren la calidad, seguridad y eficacia del producto. Asimismo, se señala que los solicitantes de medicamentos biotecnológicos biocomparables que sustenten su solicitud en un medicamento biotecnológico de referencia, deberán presentar los estudios de biocomparabilidad. (Diario Oficial de la Federación).
} 
de la UDIBI; resaltando en cada uno, la aportación a la UDIBI, y, finalmente, la cuarta sección presenta la discusión de los hallazgos identificados y la conclusión de esta investigación, así como de las líneas de investigación futuras que podrían derivarse de este estudio.

\section{La transferencia de tecnología: Elementos teóricos}

La acumulación de conocimiento útil y la extensión de sus aplicaciones representan la esencia del crecimiento moderno (Teece, 1977), el cual, se ha difundido de manera progresiva, transfiriéndose de formas muy específicas (Teece, 1975).

En este sentido, tanto las organizaciones -incluyendo aquellas instituciones que generan I\&D, se ubican en un entorno de continuos cambios tecnológicos (Bozeman, 2000), lo que les obliga a generar crear nuevos conocimientos capaces de ampliar su capacidad innovadora (Tsai, 2001) e impulsar con ello, la generación de productos innovadores con mayor valor agregado, por lo que es relevante que lo modelos de TT requeridos se adapten a las necesidades de las tecnologías actuales y futuras (Hess y Siegwart, 2013). Añadiendo a esto, la transferencia de tecnología es una importante herramienta para proponer soluciones de carácter científico y/o tecnológico (Dubickis \& Gaile-Sarkane, 2015).

Bozeman (2000) precisa que tanto la transferencia de conocimiento y de tecnología tienen una distinta connotación. La primera comprende un alcance meramente en el ámbito académico, es decir, el conocimiento científico es usado por científicos para generar o promover mayores desarrollos científicos; mientras tanto, la transferencia de tecnología, el conocimiento científico es empleado por científicos y otros en nuevas aplicaciones.

Ahora bien, a pesar de que el estudio de la transferencia de tecnología ha sido un tema que se ha venido desarrollando por más de tres décadas (Szuslanki, 1996), aún, no se cuenta con una definición generalmente aceptada por los cuerpos académicos dedicados a su análisis (Bozeman, 2000). En este sentido, se han desarrollado esfuerzos para definirla como es el caso de Argote e Ingram (2000), quienes plantean que la transferencia de tecnología es el proceso a partir del cual una unidad (grupo, departamento, o división, etc.) es influenciada por la experiencia de una u otras. Por su parte, Szuslanski (1996), expone que la TT es el intercambio diádico de conocimiento, ya que, manifiesta que, bajo sus procesos, se establecerá una relación entre la fuente proveedora y una unidad receptora. En esta misma línea, Günsel (2015) manifiesta que la transferencia de tecnología representa un constante flujo de tecnología, cuyo 
movimiento puede transcurrir de una organización a otra; incluso, expande sus alcances, porque éste puede generarse de una universidad a una organización, incluso, de un país a otro.

\section{Motivos para la generación de TT}

Los factores que motivan la transferencia de tecnología son sumamente variados pudiendo ser económicos, sociales, operacionales, estratégicas, globales e incluso, personales (Reisman, 2005) y pueden derivarse de los intereses tanto de una firma o bien, de una institución científica.

Por lo que respecta a las organizaciones, Cohen et al. (2002) consideran que la I\&D industrial tiene esencialmente dos razones para intercambiar tecnología y conocimientos están orientados hacia dos direcciones: el primero, sugiere, o bien, fomenta el desarrollo de nuevos proyectos de investigación y desarrollo; mientras que, una segunda, contribuye a complementar o finalizar proyectos existentes.

En cambio, la motivación central para la transferencia de tecnología en las instituciones, parte esencialmente de la misión institucional que tiene trazada, que es la enseñanza y la generación de ciencia básica; pero también puede establecerse a partir del interés que éstas tengan de integrarse a la dinámica de emprendedurismo institucional (Etzkowitz, 2003) y promover sus servicios científicos y/o tecnológicos al mercado (Etzkowitz \& Leydesdorff,, 2000).

\section{Mecanismos de transferencia de tecnología}

A inicios de la tercera década del siglo pasado, Schumpeter (1934) puntualizó que el desarrollo se produce a partir de la capacidad de innovación de los emprendedores, y de la introducción de nuevos métodos de producción; sin embargo, Dubickis \& Gaile-Sarkane (2015), manifiestan que si bien, es destacada la aportación de Schumpeter a la economía de la innovación, éste no precisa de manera explícita de dónde, y de forma particular, de qué mecanismos provienen estos nuevos métodos; reiterando, que la transferencia de tecnología es la que puede resolver este planteamiento.

Sobre este punto, Bayona Sáenz et al. (2003) manifiestan que las formas en que los mecanismos de TT pueden integrarse es variada, y que puede considerarse que éstas tienen una relación muy estrecha con la naturaleza de las organizaciones, sus necesidades, y particularmente, con sus estrategias. Estos mecanismos, giran alrededor de la organización, 
pudiendo ser los competidores, clientes, proveedores, consultores o firmas contratadas de investigación y desarrollo, empresas conjuntas o cooperativas, e incluso, la base de operación y fabricación propia de la firma (Cohen et al., 2002).

Por su parte, Lema y Lema (2013), distinguen dos líneas de mecanismos de TT: la primera está relacionada con el intercambio de información -esencialmente académica- y, la segunda, se orienta hacia la comercialización de la tecnología. A partir de estas dos aristas se desprenden múltiples mecanismos (ver Tabla 1).

Tabla 1. Mecanismos de transferencia de tecnología en las organizaciones

\begin{tabular}{|c|l|}
\hline \multirow{2}{*}{$\begin{array}{c}\text { Intercambio de } \\
\text { información }\end{array}$} & $\begin{array}{l}\text { Programas (sabáticos, estancias de investigación, acuerdos de colaboración) } \\
\text { Conferencias y simposio } \\
\text { Publicaciones científicas en Journals } \\
\text { Correspondencia técnica, internet / web }\end{array}$ \\
\hline \multirow{2}{*}{$\begin{array}{l}\text { Ventas y acuerdos } \\
\text { de colaboración }\end{array}$} & $\begin{array}{l}\text { Venta de equipo y/o propiedad intelectual (nuevos diseños, dibujos, bocetos, } \\
\text { encuestas de mercado). }\end{array}$ \\
& Acuerdos de colaboración (Co-producción, co-investigación, co-diseño). \\
\hline \multirow{2}{*}{ Licenciamiento } & $\begin{array}{l}\text { Licenciamiento (bocetos, diseños, manuales, data, dibujos) } \\
\text { Franquicias }\end{array}$ \\
\hline
\end{tabular}

Fuente: Elaboración propia con datos de Lema y Lema (2013)

Lema y Lema (2013), resaltan la transferencia de tecnología que se genera a partir del intercambio de información, particularmente, el que se produce dentro de la academia, reconociendo que por esta vía emerge el conocimiento científico, representando, un potencial insumo para la actividad innovadora de las organizaciones. En tanto, un segundo grupo de mecanismos, están integrados en diferentes canales como la venta de equipo o bien, a través de servicios técnicos como la consultoría, la elaboración de manuales internos, e incluso, el mantenimiento de equipo.

Finalmente, dentro de esta taxonomía, Lema y Lema (2013) colocan al licenciamiento de tecnología como un relevante eje conductor de TT, toda vez que a partir de él, pueden ser 
transmitidos conocimientos producto de la inventiva humana - ej. diseños de ingeniería, manuales de operación, software, etc.); o bien, a partir de la figura joint ventures, pueden intercambiarse, recursos o conocimientos entre dos y más actores, pero, de igual modo, empleando bajo las franquicias, se trasmite tecnología, know how y/o fórmula de servicio probada previamente.

Por lo que respecta al ámbito de las instituciones generadoras de investigación y desarrollo, el motivo central de la transferencia de tecnología es la discusión, producción, difusión y aplicación del conocimiento e innovación que den soporte al crecimiento económico (Bercovitz \& Feldman, 2006).

A partir de esta base, D'Este y Patel (2007), tras la revisión de los factores subyacentes que rodean los vínculos entre la universidad-industria y la participación del investigador en esta relación, encontraron que, de las universidades emergen diversos mecanismos de transferencia de tecnología, siendo éstos:

- Eventos y Conferencias. Asistencia a eventos promovidos por la industria, así como conferencias con la participación la industria y la academia.

- Consultoría y contratos de investigación. Encargada por la industria, sin involucrarse en la investigación inicial; por el contario, los contratos de investigación, se asientan bajo encargo, pero la investigación es iniciada y emprendida por los investigadores.

- Conformación de espacios físicos. Establecimiento de empresas spin-off; y la creación de espacios físicos con el apoyo de la industria como son laboratorios, incubadoras y centros de investigación.

- Entrenamiento. Entrenamiento de posgraduados o bien, empleados de la organización.

- Joint Research. Convenios de investigación (que involucran la investigación original y es emprendida tanto por la industria como la institución pública).

Por su parte, Cohen et al. (2002), identificaron que, sobre las actividades de I\&D de las universidades o los laboratorios o institutos gubernamentales de investigación y desarrollo, confluyen canales donde fuentes de información incluyen patentes, intercambio de información formal, publicaciones y reportes, eventos y conferencias, contratación de graduados, licencias, proyectos compartidos, contratos de investigación, consultoría e intercambio temporal personal. 
Finalmente, Radaman et al. (2017), observan que existen dos líneas de transferencia, la de tipo formal; donde se gestan mecanismos de TT como los convenios, y, por otro lado, una de carácter informal, que se desarrolla a partir de la interacción de redes informales.

\section{Metodología}

Este artículo se deriva de un caso de estudio relacionado con capacidades de absorción en la industria biofarmacéutica mexicana.

Para efectos de recolectar información empírica base para esta investigación, fueron realizadas diversas entrevistas, reconociendo que este es un método de investigación eficaz que permite recabar información relacionada con el desarrollo de un fenómeno (Yin, 1994).

Los participantes de este ejercicio metodológico tienen una relación tanto directa directivos y áreas de I\&D- como indirecta -asesores externos- en la operación de la unidad objeto de estudio que ocupa este artículo.

Durante la exploración de campo, se efectuaron veintiocho entrevistas a profundidad bajo un número importante de preguntas con carácter abierto; aunque por citar, también fueron realizadas algunas bajo una estructura cerrada para que fueran respondidas bajo una escala Likert.

Por otra parte, para llevar a cabo este ejercicio metodológico, se emplearon cuestionarios estructurados de acuerdo al tipo de actividad de cada grupo de entrevistados y la función que ejercen dentro de la organización en estudio. Es decir, para el personal de las áreas de investigación, los cuestionarios fueron formulados con la finalidad de identificar todas aquellas fuentes de conocimiento que convergen dentro de la UDIBI y la forma en cómo éstas influyen, no sólo en la ejecución de su actividad, sino lo que éstas aportan al conocimiento propio. Por lo que respecta a la directiva, las entrevistas estaban orientadas a conocer las estrategias - presentes y futuras- para allegarse de conocimiento complementario y la forma en cómo operan éstas y los actores -públicos o privados- que las integran.

En relación a los cuestionarios formulados para aquellos participantes con una actividad indirecta, -ej. asesores externos, las preguntas fueron formuladas sobre los conductos por los cuales éstos proveen conocimiento y, en qué productos, éste se puede materializar y servir de base al proceso de asimilación de la UDIBI. 
De igual manera, fue considerado como elemento metodológico, la consulta y la revisión -bajo determinados alcances de confidencialidad- algunos de los convenios de colaboración que mantiene el laboratorio politécnico en estudio con actores externos de la industria biofarmacéutica y biotecnológica.

Para efectos de este artículo, tanto en el proceso de obtención de la data como de su procesamiento, fueron observados todos y cada uno de los canales de TT que confluyen en la UDIBI, y, por tanto, se consideró pertinente, resaltar su importancia y difundir los hallazgos identificados.

Para efectos posteriores, el procesamiento de la información contenida en las entrevistas comprendió la transcripción, clasificación a partir del empleo del software NVIVO. Una vez realizada esta actividad, la data fue analizada y empleada para el transcurso de la investigación citada, como para el desarrollo de otros trabajos empíricos.

\section{Mecanismos de transferencia de tecnología en la UDIBI}

Las diferentes fases de desarrollo de capacidades de la UDIBI le han hecho conformar una sólida estructura de conocimiento acumulado que sirve, como lo demarcan Cohen y Levinthal (1990), de insumo para sus operaciones críticas de I\&D. Pero ésta, no sólo se ha conformado a partir de sus propios recursos, sino de la interacción con otros actores, quienes, a partir del intercambio de conocimiento y experiencias, la han permitido incrementar y robustecer dicha base.

Los mecanismos de transferencia de tecnología que confluyen dentro de este laboratorio institucional son diversos y con alcances diferentes, dependiendo, en gran medida de las características de cada actor o fuente de conocimiento.

Las características de la UDIBI descritas en la parte introductoria, hacen resaltar las dos líneas de mecanismos de transferencia de tecnología, es decir, los de carácter organizacional y los de tipo institucional, reflejándose en conjunto de la siguiente manera:

\section{a) Mecanismos de TT de carácter organizacional}

\section{Colaboración con socios estratégicos}

De acuerdo con información recopilada en entrevistas, en México, el mercado de los servicios tecnológicos, particularmente los relacionados a los análisis de biocomparabilidad de 
medicamentos biotecnológicos, se encuentra en una fase de reordenamiento, ya que se están incorporado un mayor número de proveedores de este tipo de servicios, lo que acota las oportunidades de crecimiento para los que actualmente los realizan.

Ante este escenario, la UDIBI ha reformulado estrategias para diversificar su oferta comercial y generar otros productos tecnológicos de interés de la biofarmacéutica. Para llevar a cabo este propósito, ha establecido convenios de colaboración inter-organizacional con otros actores integrantes de la cadena de valor de la IBF. -ej. empresas de biotecnología- con la finalidad de desarrollar proyectos de I\&D. De esta forma, además de integrarse al desarrollo de nuevos proyectos científicos, obtiene conocimientos similares y/o complementarios.

\section{Licenciamiento de Tecnología}

La UDIBI, desde hace aproximadamente tres años, mantiene una estrecha colaboración con una empresa de biotecnología de Estados Unidos, dedicada al diseño de anticuerpos para el desarrollo de tratamientos médicos. El proyecto en conjunto está orientado a la conformación de una biblioteca de anticuerpos desplegada en "fagos" o Phage Display (como se le conoce en inglés).

En este proyecto, la UDIBI, brinda servicios tecnológicos desarrollando los métodos y la implementación de tecnologías, apegándose estrictamente a los criterios, requisitos y necesidades establecidos en el contrato de servicios y al licenciamiento de tecnología concedido por la empresa estadounidense.

Los beneficios que trae consigo este mecanismo de TT para este laboratorio institucional son múltiples y con alcances distintos, ya que no sólo se ha apropiado del conocimiento externo a partir de la absorción, sino que también, ha generado conocimiento propio a partir de la configuración de métodos, búsqueda bibliográfica, desarrollo e implementación de procesos, lo que ha permitido incursionar en una línea de investigación nueva con perspectivas comerciales atractivas y relevantes para sus objetivos. Es por tanto, que esta firma estadounidense, se ha convertido en un factor clave y estratégico para sus fines científicos y tecnológicos.

\section{Otras instituciones y organizaciones externas al IPN}

Los motivos que hacen a la UDIBI establecer contacto con otras IES, es porque en ocasiones, para el desarrollo y ejecución de ciertos proyectos, la primera no cuenta; ya sea con el 
conocimiento, el equipo y/o infraestructura para llevarlos a cabo. De esta manera, y con el objeto de dar solución a esta problemática, recurre a laboratorios o unidades científicas incorporados en otras universidades o centros de investigación externos al IPN, tales son los casos de la Universidad Nacional Autónoma de México (UNAM), el Centro de Investigación y Estudios Avanzados del IPN (CINVESTAV), entre otros.

Por otra parte, otra figura que también puede representar un canal de transferencia es la terciarización de servicios tecnológicos, misma que se presenta cuando la UDIBI no cuenta (de forma parcial o total) con el conocimiento, experiencia $y / o$ infraestructura y equipos para desarrollarlo por cuenta propia; por lo que, se acerca a otra organización intermedia OI, para que, ésta última realice el servicio de forma parcial (o, en su caso total), dando pie, a que este laboratorio se allegue de conocimiento de manera directa o indirecta, lo cual es útil para nutrir la plataforma de su propio conocimiento.

Este mecanismo de transferencia de tecnología permite tanto a la UDIBI, como a las partes involucradas generar y/o allegarse de nuevo conocimiento científico y técnico, a partir de la formulación o el planteamiento de nuevos criterios y /o metodologías para el manejo de animales de laboratorio, equipos especializados, gestión de moléculas y cuerpos celulares, etcétera, lo que contribuye a generar y/o acrecentar la experiencia y las habilidades tecnológicas.

Es conveniente resaltar que, entre la colaboración académica, no existe un intercambio económico de por medio. El beneficio que la UDIBI llega a obtener, es el reconocimiento y/o agradecimiento a ésta, o bien, la participación del cuerpo de investigadores en trabajos de investigación, artículos científicos, publicaciones de libros, etcétera.

\section{Clientes}

Los clientes de la industria biofarmacéutica no sólo representan una fuente de ingresos para la UDIBI, sino que también, de acuerdo a los entrevistados, significan una fuente de la inventiva.

A partir de la exposición de las características del producto y necesidades y problemáticas determinadas por parte de los clientes, el equipo de investigación de la UDIBI diseña y conforma protocolos y metodologías científicas para dar solución específica a problemas determinados dentro de un proyecto o desarrollo que los primeros demanden.

Por otra parte, el efecto de la transferencia no sólo se produce de los clientes hacia la UDIBI; sino viceversa, toda vez que esta última les brinda asesoría sobre el marco legal aplicado a la 
biofarmacéutica en México y la forma en cómo deben ser formulados los reportes que demanda la autoridad sanitaria mexicana, así como, el contenido de guías biofarmacéuticas vigentes en Estados Unidos, la Unión Europea y México.

\section{Proveedores}

Los proveedores de la UDIBI forman también parte importante de los hallazgos relacionados con mecanismos de transferencia de tecnología.

Por la función de abastecer insumos y proveer servicios a la UDIBI, pero, en particular, por el contacto directo con el mercado, los proveedores de esta organización, representan una vía de TT de vital importancia, ya que además de conocimiento, transmite experiencia y habilidades que se reproducen de forma intra-organizacional (Ortíz, 2016).

La gran parte de las ocasiones, el conocimiento que brinda el proveedor, está soportado por la preparación técnica previa que tiene el personal de la UDIBI. Lo anterior se explica porque el proveedor asume que el conocimiento base para la operación de un determinado equipo, insumo, o reactivo "ya está dado" por parte de la organización, lo que representa un reto extra al requerir contratar y/o capacitar a un especialista para la operación, principalmente cuando se trata de equipos.

\section{Asesores externos}

La UDIBI cuenta con un grupo de consultores altamente especializados en materia farmacéutica e inmunológica. Dichos consultores son en sí, científicos reconocidos a nivel nacional e internacional.

En algunos casos, algunos de estos consultores estuvieron presentes en los procesos de conformación de la UDIBI, lo que significa que, por su parte, existe un conocimiento de esta unidad científica, así como de sus metas y estrategias. Bajo esta perspectiva, este grupo de expertos emite sus opiniones y/o recomendaciones en torno al desarrollo de proyectos de I\&D así como la solución de problemas específicos.

Como producto de la estrecha colaboración que existe entre este grupo de especialistas y el equipo de investigadores de la UDIBI, se desprenden trabajos de investigación que han llegado a convertirse en tesis; o bien, de difusión científica como artículos y ponencias, mismas que han sido expuestas en distintos foros y/o congresos nacionales e internacionales. 


\section{Convenios de colaboración}

El laboratorio institucional en referencia formaliza los servicios que ofrece a la industria privada a través de convenios de colaboración.

Dentro de la UDIBI tienen dos tipos de convenios, los que sostienen un proyecto en curso, y los que ya fueron entregados los resultados de un servicio, y no se ha agotado el tiempo del término del contrato; esto, con el fin de solventar algún aspecto técnico o retroalimentación con el cliente.

Al momento de la realización del estudio de campo, la unidad objeto de estudio, la gran mayoría de los convenios en curso estaban signados tanto con empresas farmacéuticas mexicanas de capital extranjero, como con aquellas de corte nacional, con el objetivo de analizar y hacer pruebas de biocomparabilidad en distintas moléculas. De igual manera, se contaba con tanto con convenio para realizar pruebas preclínicas para una empresa farmacéutica de capital nacional para el tratamiento de distintas enfermedades (humanas y veterinarias) y con otro, con una empresa de biotecnología de Estados Unidos para conformación de una biblioteca de anticuerpos.

\section{b) Mecanismos de TT de carácter institucional}

Bajo el entorno institucional que imprime el Instituto Politécnico, también se generan mecanismos de transferencia útiles tanto para la propia institución educativa como para la interacción e intercambio de conocimiento entre la UDIBI y la industria. Tales mecanismos son:

\section{Bases de datos especializadas}

El desarrollo de la ciencia básica formulada en la UDIBI, está soportada por la revisión del estado del arte, actividad que se convierte en crítica y vital dada su relevancia, toda vez que representa la base de análisis de las diferentes metodologías, protocolos de evaluación para el tratamiento de diversos tipos de moléculas, cuerpos celulares, etcétera.

Lo anterior representa, como lo señaló uno de los científicos entrevistados, una fuente de conocimiento constante y actual, que permite; no sólo conocer los avances en ciencia farmacológica, inmunológica y patológica de frontera, sino también, consolidar un sólido 
conocimiento teórico, e identificar, las áreas de oportunidad y potenciales soluciones a las problemáticas expuestas por sus clientes.

Tras la revisión de esta literatura, los hallazgos más relevantes son consultados entre los miembros que integran un proyecto de investigación y, tras su discusión y conclusiones, son puestos en práctica para la realización del mismo.

\section{Capacitación}

En su totalidad, los distintos entrevistados manifestaron que una de las fortalezas que sostiene a la UDIBI es el alto nivel de experiencia y conocimiento de su capital humano.

El personal que integra este laboratorio científico está integrado un grupo de investigadores de alta y sólida capacidad tecno-científica cuyas áreas de conocimiento son, entre otras, la inmunología, biomedicina molecular, farmacología; asimismo, tienen especializaciones muy definidas como el manejo de equipos especializados o bien, la realización de ensayos y múltiples pruebas de laboratorio (ej. diseño y estandarización de pruebas en biofarmacéuticos, pruebas preclínicas, etcétera).

De acuerdo a la información recopilada, al interior de la UDIBI, la formación del recurso humano, se desarrolla bajo estas tres líneas:

a) Capacitación específica: El personal que se integra a la UDIBI, si bien cuenta con conocimientos científicos esenciales, es capacitado para encaminarlo al perfil que se desea y éste desarrolle actividades específicas de acuerdo a las necesidades de la organización; pero también, un mayor potencial, atendiendo a sus habilidades y competencias.

b) Talleres internos. A manera de compartir el conocimiento adquirido en foros académicos, se generan de manera interna talleres para exponer los puntos más trascendentes y actuales en las diferentes líneas de conocimiento.

c) Congresos nacionales e internacionales. El personal que integra el grupo de investigación de la organización en estudio, es capacitado vía congresos académicos nacionales y, de forma más constante, acude a congresos organizados por los más destacados colegios disciplinarios a nivel internacional. La gran mayoría del personal asisten o han asistido a este tipo de foros como mínimo dos veces por año, la gran 
mayoría de las veces como ponentes de alguna investigación surgida de algún proyecto realizado en la UDIBI, y, en algunas ocasiones como asistentes. como ponentes

Este mecanismo de aprendizaje contribuye a que el personal se instale en las técnicas y bases científicas más actuales de acuerdo a su línea de investigación.

\section{Equipos de investigación internos}

De manera formal y de manera recurrente (semanalmente) los distintos equipos de trabajo de la UDIBI mantienen relaciones de trabajo, en la que se discute, las diferentes estrategias de solución ante potenciales problemáticas, o bien, el seguimiento de resultados de los diferentes proyectos o servicios tecnológicos en gestión. Estas reuniones, se llevan a cabo con el Comité Científico, que, por ley, debe estar instaurado con científicos altamente especializados en materia farmacéutica e inmunológica.

Por otra parte, la línea de intercambio de conocimiento que tiene un mayor acento, son los mecanismos informales de interacción entre los mismos miembros de los grupos de trabajo. Bajo este contexto, se buscan y propone la revisión de artículos científicos, la ejecución de determinadas metodologías, la consulta con otros pares científicos de IES externas al IPN, o bien, el apoyo para la ejecución de pruebas y ensayos; esto marca, sin duda, un vínculo estrecho de conocimiento que no se instaura de manera formal, pero que genera un importante flujo de conocimiento y experiencia intra-organizacional.

\section{Artículos científicos}

El conocimiento derivado de la labor científica desarrollada en los proyectos tanto por el equipo de científicos pertenecientes a la UDIBI, sean de carácter interno (esencialmente alrededor del Transferón ${ }^{\circledR}$ ), o bien, del trabajo de investigación por parte de los alumnos en estancia, se traduce, en una múltiple variedad de artículos científicos publicados en diferentes journals (ej. Journal of Medicinal Food, International Journal of Molecular Science, de las especialidades inmunología, Journal of Immunology Research, etc. $)^{4}$.

\footnotetext{
${ }^{4}$ Algunos de estos artículos son: Salinas-Jazmín, N., Estrada-Parra, S., Becerril-García, M. A., Limón-Flores, A. Y., Vázquez-Leyva, S., Medina-Rivero, E., \& Pérez-Tapia, S. M. (2015). Herpes murine model as a biological assay to test dialyzable leukocyte extracts activity. Journal of immunology research, 2015; Santacruz, C., Linares, M., Garfias, Y., Loustaunau, L. M., Pavón, L., Perez-Tapia, S. M., \& Jimenez-Martinez, M. C. (2015). Expression of IL-
} 
Los artículos científicos constituyen un mecanismo de difusión de conocimiento que no sólo es empleado en la academia, sino también, aunque sea, en una menor proporción en la industria privada. Con relación a este último punto y de acuerdo con algunos de los entrevistados en este estudio, en las reuniones técnicas que se sostienen con los clientes, los investigadores a cargo de los proyectos, sugieren al personal de las organizaciones la revisión de documentos científicos, con el fin de homogenizar conocimiento y establecer un marco de entendimiento común para la solución de una problemática dada.

\section{Dependencias politécnicas vinculantes con la industria}

Bajo el marco regulatorio que envuelve al IPN, esta IES publica ${ }^{5}$, tiene establecido el estimular la transferencia de tecnología, proyectos de investigación, estudios técnicos, asesoría, normalización, capacitación de recursos humanos, así como la certificación de calidad que favorezcan la productividad, competitividad e innovación en las empresas y otras organizaciones públicas.

Para tal efecto, fue creada la Unidad Politécnica para el Desarrollo y la Competitividad Empresarial (UPDCE), se constituye como una dependencia politécnica que tiene como objetivos vincular la actividad científica y tecnológica del IPN con el sector industrial nacional y contribuir en el desarrollo de la competitividad empresarial nacional al ofrecer servicios de gestión, consultoría, formación, capacitación, transferencia y comercialización de tecnología y de servicios tecnológicos (IPN, s.f.).

\section{Estancia de estudiantes}

Para la UDIBI, las estancias de estudiantes representan, en una primera instancia, al desarrollar sus proyectos de investigación (nivel licenciatura y posgrado), aportan a los diferentes grupos de investigación conocimientos teóricos y técnicas nuevas, así como habilidades; mientras que, por otro lado, contribuyen al curso de los distintos proyectos científicos desarrollados por esta unidad científica.

\footnotetext{
${ }^{8}$ IL- 6 and IL-1 $\beta$ in tears as a main characteristic of the immune response in human microbial keratitis. International Journal of Molecular Sciences, 16(3), 4850-4864.

${ }^{5}$ Reglamento de Integración Social del Instituto Politécnico Nacional
} 


\section{Servicios de Consultoría}

La UDIBI ofrece servicios de consultoría a la industria farmacéutica biofarmacéutica relacionada a los múltiples aspectos que debe cumplir en materia regulatoria, con el fin de obtener la certificación de biocomparabilidad en medicamentos biosimilares ${ }^{6}$ y lograr su comercialización en el interior del país.

\section{Comités Institucionales}

El IPN es considerado como uno de los actores de relevancia en el desarrollo de ciencia básica y desarrollos tecnológicos en México; por tanto, la participación y consulta en foros y/o comités, es un obligado recurso con el que cuentan instituciones públicas y/o privadas para el desarrollo e implementación de políticas públicas sectoriales.

En este caso, en representación de esta institución educativa, la UDIBI, forma parte de los distintos comités que organiza la COFEPRIS, para, proponer, conformar e implementar de normas sanitarias, o bien, revisar la legislación sanitaria vigente. Este importante hecho, no sólo convierte a esta organización politécnica en un actor participante de la política pública sanitaria, sino que, también le permite contar con información de vanguardia relacionada a las tendencias y avances tecnológicos mundiales, lo que le permite definir estrategias no sólo para el desarrollo de nuevos proyectos o la reorientación de los mismos a nivel interno; sino, representa la oportunidad diseñar potenciales colaboraciones o bien, robustecer las existentes.

Hasta aquí, el gran conjunto de mecanismos de TT que confluyen hacia la UDIBI permite observar que el conocimiento provisto por estas fuentes -sean del enfoque organizacional o institucional-, potencian sus esfuerzos en la generación de ciencia básica y desarrollos tecnológicos ahí desarrollados; pero, por otra parte, se aprecia que no sólo ésta recibe conocimiento; sino que también, la UDIBI contribuye al conocimiento de dichos actores o fuentes, convirtiendo este flujo en bidireccional (ver ilustración 1).

\footnotetext{
${ }^{6}$ Un biosimilar es un producto biológico que es aprobado por mostrar alta similitud con el producto biológico de referencia (FDA, 2016)
} 
Ilustración 1. Mecanismos de transferencias de Tecnología de la UDIBI y el sentido del flujo de conocimiento

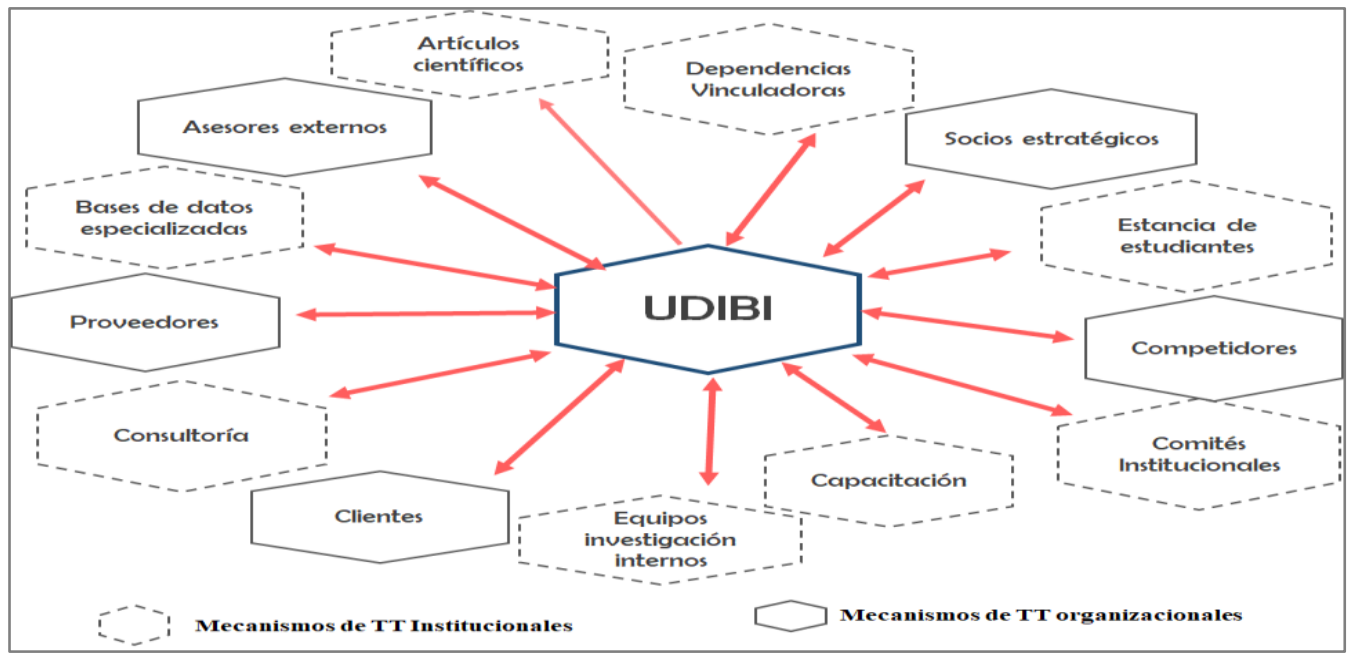

Fuente: Elaboración propia

Considerando el argumento anterior, puede considerarse a la UDIBI como un mecanismo de transferencia de tecnología, al contribuir a la conformación del conocimiento de sus propias fuentes. Algunos ejemplos de esta relación y contenido pueden citarse de la siguiente manera:

- UDIBI - Proveedores: Proporciona elementos científicos de frontera y tendencias tecnológicas útiles para que los proveedores identifiquen y le ofrezcan mejores alternativas en bienes y servicios.

- UDIBI - Clientes: Ante una problemática específica, la UDIBI proporciona elementos científicos orientados a ofrecer una potencial solución tanto de forma previa, como durante la gestión del servicio.

- UDIBI- Unidades científicas IPN. La UDIBI brinda apoyo a otras dependencias científicas para la realización de pruebas, ensayos y uso de equipos especializados, compartiendo con colegas conocimiento y habilidades técnicas.

- UDIBI-Capacitación (Congresos). La asistencia de investigadores a foros académicos permite transmitir las experiencias y hallazgos científicos encontrados en el desarrollo de proyectos científicos ahí realizados. 


\section{Discusión y Conclusiones}

Las organizaciones, particularmente, aquellas que desarrollan su actividad innovadora en tecnologías de alta intensidad tecnológica, deben, de forma indispensable, adquirir conocimiento de terceros (Yoo et al., 2015).

Considerando este argumento, el conocimiento que se desarrolla en la UDIBI parte de diversos mecanismos de TT que se alinean a las características propuestas por Günsel (2015), apreciando que éstos, se generan al intercambiar y/o compartir conocimiento similar o complementario, sea, desde el interior de una firma o bien, de un grupo interdisciplinario.

Si bien el pertenecer a una IES pública en México, hace a la unidad científica aquí estudiada, apegarse a un marco legal y de procesamientos altamente institucionalizado y rígido, ésta, coincidiendo con Lichtenthaler \& Lichtenthaler (2010), mantiene a su interior, un sistema administrativo flexible orientado no sólo a la innovación abierta, que la impulsa a colaborar con diversos actores externos para adquirir conocimientos y tecnología que complementen, como lo manifiestan, Nelson y Winter (1982) y Einsenhardt y Martin (2000) la I\&D y recursos internos. Basado en lo anterior, durante el desarrollo de este estudio se encontró que, detrás de los vínculos de colaboración entre la UDIBI y otros actores, existe una cantidad significativa, pero en particular, una variada combinación de mecanismos -organizacionales e institucionales-, como señala Cohen et al. (2000) para transferir tecnología, lo que también brinda, un amplio abanico de conocimientos para ser apropiados a su interior.

\section{Conclusiones}

Los mecanismos de transferencia de tecnología observados en este laboratorio politécnico guardan una estrecha relación con las capacidades tecnológicas ahí desarrolladas, mismas que han acompañado, su evolución y expansión, en el sentido de requerir conocimiento proveniente de fuentes externas para fortalecerlas. Todos, en su conjunto, permiten un adecuado engranaje para el desarrollo de sus operaciones crítica.

De acuerdo a su tipo, los distintos mecanismos de TT que actúan en la UDIBI desarrollan un rol sumamente relevante. Por tratarse de una organización intermedia enclavada en la cadena de valor de la industria biofarmacéutica, los canales de transferencia de carácter organizacional, le permiten mantener un estrecho contacto con necesidades puntuales del sector (clientes), contar con el conocimiento de tendencias de mercado (proveedores), y, de acuerdo al 
caso, entablar redes de colaboración con otros actores para el desarrollo de servicios tecnológicos (socios estratégicos).

Por otra parte, los mecanismos de tipo institucional, contribuyen a reafirmar no sólo la misión científica y de formación para la que fue creada, sino que también, a través de éstos, la UDIBI, corrobora la relevancia e influencia en la vida científica, industrial y social del país, al participar de forma activa en la instauración y revisión de la política pública sanitaria nacional. En suma, todos estos hallazgos, más otros, identificados a lo largo del estudio de caso, permiten señalar que, la actividad de la UDIBI enarbola un gran significado para el país. Por una parte, ante la ausencia de organizaciones privadas con las suficientes capacidades científicas y tecnológicas en la biofarmacéutica mexicana, este laboratorio surge como un agente, capaz de cubrir, con servicios de alto estándar científico, acuciosas demandas de este sector. Mientras que, por otro lado, contribuye a engrandecer la actividad científica del IPN, al ser parte fundamental e integral del desarrollo de un biofármaco, cuyos efectos mejoran la salud y calidad de vida de sus consumidores.

Lo anterior hace a la UDIBI uno de los escasos casos de excepción en unidades científicas integradas a IES públicas en México, no sólo por autosustentar su operación con ingresos autogenerados, sino que, a través de una visión y estrategias de corte científicocomercial, perfila su acción hacia los parámetros de la universidad emprendedora.

\section{$\underline{\text { Agradecimientos }}$}

Se agradece al Instituto Politécnico Nacional, en particular a la directiva y personal de la UDIBI, por la disposición y apoyo para la realización de este artículo.

\section{$\underline{\text { Referencias }}$}

Argote, L. I. (2000). Knowledge transfer in organizations: Learning from the experience of others. Organizational behavior and human decision processes, 82(1), 1-8.

Argote, L., \& Ingram, P. (2000). Knowledge transfer: A basis for competitive advantage in firms. Organizational behavior and human decision processes, 82(1), 150-169.

Arora, A., Fosfuri, A., \& Gambardella, A. (2004). A.Markets for technology: The economics of innovation and corporate strategy. MIT press.

Audretsch, D. B. (2001). The role of small firms in US biotechnology clusters. Small Business 
Mecanismos de transferencia de tecnología como elementos del fortalecimiento del conocimiento acumulado en la industria biofarmacéutica mexicana: El Caso de la UDIBI - IPN

Economics, 17((1-2)), 3-15.

Audretsch, D., \& Feldman, M. (2003). Small-firm strategic research partnerships: The case of biotechnology. 15(2),. Technology analysis \& strategic management, 2, 273-288.

Bayona Sáez, C., García-Marco, T., \& HUERTA ARRIBAS, E. (2003). ¿ Cooperar en I+ D? Con quién y para qué. Revista de Economía Aplicada, 11(31).

Berbegal-Mirabent, J., Alegre, I., \& Guerrero, A. (2017). University Research Parks and their impact on technology transfer activities. Technological Innovation Networks: Collaboration and Partnership (Vol. 167).

Bercovitz, J., \& Feldman, M. (2006). Entpreprenerial universities and technology transfer: A conceptual framework for understanding knowledge-based economic development. The Journal of Technology Transfer, 31(1).

Bianchi, M., \& et al. (2011). Organisational modes for Open Innovation in the biopharmaceutical industry: An exploratory analysis,. Technovation, 31(1), 22-33.

Bozeman, B. (2000). Technology transfer and public policy: a review of research and theory. Research Policy, 627-655.

Bozeman, B., \& et al. (2015). The evolving state-of-the-art in technology transfer research: Revisiting the contingent effectiveness model. Research Policy, 44(1), 34-49.

Chesbrough, H., \& Crowther, A. (2006). Beyond high tech: early adopters of open innovation in other industries. $R \& D$ Management, 36(3), 229-236.

Cohen, W., \& Levin, R. (1989). Empirical studies of innovation and market structure. Handbook of industrial organization, 2, 1059-1107.

Cohen, W., \& Levinthal, D. (1990). Absorptive capacity: A new perspective on learning and innovation. Strategic Learning in a Knowledge economy, 39-67.

Cohen, W., Nelson, R., \& Walsh, J. (2002). Links and impacts: the influence of public research on industrial R\&D. Management science, 48(1), 1-23.

D'Este, P., \& Patel, P. (2007). University-industry linkages in the UK: What are the factors underlying the variety of interactions with industry? Research policy, 36(9), 1295-1313.

Debackere, K., \& Veugelers, R. (2005). The role of academic technology transfer organizations in improving industry science links. Research policy, 34,(3), 321-342.

Diario Oficial de la Federación . (2014). NORMA Oficial Mexicana NOM-257-SSA1-2014 en materia de medicamentos biotecnológicos. 
Dubickis, M., \& Gaile-Sarkane, E. (2015). Perspectives on Innovation and Technology Transfer. Procedia-Social and Behavioral Science, 213, 965-970.

Eisenhardt, K., \& Martin, J. (2000). Dynamic capabilities: what are they? Strategic management journal, 21(10-11), 1105-1121.

Etzkowitz, H. (2003). Innovation in innovation: The triple helix of university-industrygovernment relations. Social science information, 42(3), 293-337.

Etzkowitz, H. (2003). Research groups as 'quasi-firms': the invention of the entrepreneurial university. Research Policy, 32, 109-121.

Etzkowitz, H., \& et al. (2000). The future of the university and the university of the future: evolution of ivory tower to entrepreneurial paradigm. Research policy, 29(2), 313-330.

Etzkowitz, H., \& Leydesdorff, L. (2000). The dynamics of innovation: from National Systems and "Mode 2" to a Triple Helix of university-industry-government relations. Research policy, 2, 109-123.

Etzkowitz, H., \& Leydesdorff,, L. (2000). The dynamics of innovation: from National Systems and "Mode 2" to a Triple Helix of university-industry-government relations. Research policy, 29(2), 109-123.

Fukugawa, N. (2016). Knowledge spillover from university research before the national innovation system reform in Japan: localisation, mechanisms, and intermediaries. Asian Journal of Technology Innovation, 24(1), 100-122.

Günsel, A. (2015). Research on effectiveness of technology transfer from a knowledge based perspective. Procedia-Social and Behavioral Sciences, 777-785.

Guzman, A. (2014). Propiedad intelectual y capacidades de innovación en la industria farmacéutica de Argentina, Brasil y México. Mexico: UAM-Gedisa editorial .

Heller, M., \& y Eisenberg, R. (1998). Can patents deter innovation? The anticommons in biomedical research. Science,, 698-701.

Hess, S., \& Siegwart, R. (2013 ). R\&D Venture: proposition of a technology transfer concept for breakthrough technologies with R\&D cooperation: A case study in the energy sector. 38(2),. The Journal of Technology Transfer, 153-179.

Hu, A., Jefferson, G., \& Jinchang, Q. (2005). R\&D and technology transfer: firm-level evidence from Chinese industry. ,. Review of Economics and Statistics, 87(4), 780-786.

IPN. (n.d.). UPDCE . Retrieved from http://www.updce.ipn.mx/Paginas/inicio.aspx 
Mecanismos de transferencia de tecnología como elementos del fortalecimiento del conocimiento acumulado en la industria biofarmacéutica mexicana: El Caso de la UDIBI - IPN

Lema, A., \& Lema, R. (2013). Technology transfer in the clean development mechanism: Insights from wind power. Global Environmental Change, 23(1), 301-313.

Levin, R., Klevorick, A., Nelson, R., \& Winter, S. (1987). Appropriating the returns from industrial research and development. Brookings papers on economic activity, 3, 783-831.

Levitt, B., \& March, J. (1988). Organizational learning. Annual Review of Sociology,, 14, 319340.

Lichtenthaler, U., \& Lichtenthaler, E. (2010). Technology transfer across organizational boundaries: absorptive capacity and desorptive capacity. California Management Review, 53(1), 154-170.

Mowery, D., \& Oxley, J. (1995). Inward technology transfer and competitiveness: the role of national innovation systems. Cambridge journal of economics, 19(1), 67-93.

Nelson, R., \& Winter, S. (1982). An evolutionary theory of economic change.

Ortiz, A. (2016). Capacidades Tecnológicas y de vinculación: Unidad de Desarrollo e Investigación en Bioprocesos para servicios la industria biofarmacéutica. Tesis de Maestria, Instituto Politécnico Nacional.

Pérez, P. (2014). Organizaciones intermedias en la actividad innovadora: el caso de México. . México: Instituto Politécnico Nacional.

Perkmann, M., \& Walsh, K. (2007). University-industry relationships and open innovation: Towards a research agenda. International Journal of Management Reviews, 9(4), 259280.

Powell, W. (1998). Learning from collaboration: Knowledge and networks in the biotechnology and pharmaceutical industries. California management review, 40(3), 228-240.

Ramadhan, F. S. (2017). Mechanisms for effective tacit knowledge transfer in university laboratory: An agent-based approach. . In 2017 IEEE International Conference on Industrial Engineering and Engineering Management (IEEM), (pp. 1138-1142).

Reisman, A. (2005). Transfer of technologies: a cross-disciplinary taxonomy. Omega, 33 (3), 189-202.

Rothaermel,, F. (2001). Complementary assets, strategic alliances, and the incumbent's advantage: an empirical study of industry and firm effects in the biopharmaceutical industry. Research policy, 30(8), 1235-1251.

Schumpeter, A. ((1943) 2010). Capitalism, socialism and democracy. Routledge. 
Szulanski, G. (1996). Exploring internal stickiness: Impediments to the transfer of best practice within the firm.,. Strategic management journal, 17(S2), 27-43.

Teece, D. (1975). Multinational corporation and the resource cost of international technology transfer. Pennsylvania: University of Pennsylvania.

Teece, D. (1977). Technology transfer by multinational firms: the resoursce cost of transfering Techological know-how. The Economic Journal, 242-261.

Teece, D., \& et al. (1997). Dynamic capabilities and strategic management. Strategic management journal, 18(7), 509-533.

Tsai, W. (2001). Knowledge transfer in intraorganizational networks: Effects of network position and absorptive capacity on business unit innovation and performance. Academy of management journal, 44(5), 996-1004.

UDIMEB-IPN. (n.d.). Retrieved from http://www.udimeb.ipn.mx/

Yin, R. (1994). Case study research and applications: Design and methods. . Sage publications.

Yoo, S., Sawyer, O., \& Tan, W. (2015). The impact of exogenous and endogenous factors on external knowledge sourcing for innovation: The dual effects of the external environment. The Journal of High Technology Management Research, 26(1), 14-26. 